\title{
天然有机质在生物炭负载纳米镍铁降解十溴联苯醚过程中的影响作用 机理辨识
}

\author{
易云强 $a, b, c$ 吴娟 $a, b, c$ 方战强 $*, a, b, c$ \\ ( ${ }^{a}$ 华南师范大学 化学与环境学院 广州 510006) \\ ( ${ }^{b}$ 广东省水环境生态治理与修复工程技术研究中心 广州 510006) \\ ( ${ }^{c}$ 广东高校城市水环境生态治理与修复工程技术研究中心 广州 510006)
}

\begin{abstract}
摘要 天然有机质(NOM)对纳米铁基材料去除污染物过程中的影响作用机理仍存争议. 本工作选取腐殖酸(HA)作为 $\mathrm{NOM}$ 的代表物, 研究了 HA 对生物炭负载纳米镍铁 $(\mathrm{BC} @ \mathrm{Ni} / \mathrm{Fe})$ 降解十溴联苯醚(BDE209)的动力学影响, 结果表明 HA 对 BC@Ni/Fe 降解 BDE209 产生抑制作用, 且随着 HA 浓度的升高, 抑制作用越显著. 通过 HA 与 BC@ $\mathrm{Ni} / \mathrm{Fe}$ 相互作用 实验发现, HA 能够快速地被 $\mathrm{BC} @ \mathrm{Ni} / \mathrm{Fe}$ 吸附; 通过 HA 对 $\mathrm{BC} @ \mathrm{Ni} / \mathrm{Fe}$ 的 Zeta 电位和沉降影响实验发现, 随着 HA 浓度 的升高可有效提高 $\mathrm{BC} @ \mathrm{Ni} / \mathrm{Fe}$ 的稳定性以及表面电荷, 这表明 $\mathrm{HA}$ 不是通过影响 $\mathrm{BC} @ \mathrm{Ni} / \mathrm{Fe}$ 颗粒的性能对 $\mathrm{BC} @ \mathrm{Ni} / \mathrm{Fe}$ 去除 BDE209产生抑制作用的. BC@Ni/Fe 的腐蚀能力随着 HA 浓度的升高而降低, 这与 HA 对 BC@Ni/Fe 去除 BDE209 反应活性影响成正相关关系. HA 中具有电子传递作用的典型醌类化合物 2-羟基-1,4-菜醌(Lawsone)和蒽醌-2,6-磺酸钠 (AQDS), 在反应过程中并没有起到电子传递作用促进反应进行, 反而对 BC@ $\mathrm{Ni} / \mathrm{Fe}$ 去除 BDE209 起抑制作用. 结合 BDE209 和 HA 之间的竞争吸附实验发现, HA 抑制 BC@Ni/Fe 反应活性的主导原因是 $\mathrm{HA}$ 优先于 BDE209 被 BC@ Ni/Fe 吸附, $\mathrm{HA}$ 包覆于 $\mathrm{BC} @ \mathrm{Ni} / \mathrm{Fe}$ 颗粒表面占据了活性位点, 阻碍 $\mathrm{BC} @ \mathrm{Ni} / \mathrm{Fe}$ 与 $\mathrm{H}_{2} \mathrm{O}$ 的接触, 减少了 $\mathrm{Fe}^{0}$ 的腐蚀, 从而抑制 了 BC@ Ni/Fe 对 BDE209 的降解.
\end{abstract}

关键词 天然有机质; 纳米零价铁; 生物炭负载纳米镍铁; 十溴联苯醚; 影响机制

\section{Identification Influence Mechanism of Humic Acid in the Degradation of Decabromodiphenyl Ether by the BC@Ni/Fe Nanoparticles}

\author{
Yi, Yunqiang ${ }^{a, b, c}$ \\ $\mathrm{Wu}, \mathrm{Juan}^{a, b, c}$ \\ Fang, Zhanqiang ${ }^{*, a, b, c}$
}

( ${ }^{a}$ School of Chemistry and Environment, South China Normal University, Guangzhou 510006)

( ${ }^{b}$ Guangdong Technology Research Centre for Ecological Management and Remediation of Water System, Guangzhou 510006)

( ${ }^{c}$ Guangdong Technology Research Centre for Ecological Management and Remediation of Urban Water System, Guangzhou 510006)

\begin{abstract}
The influence mechanism of natural organic matter (NOM) on the removal of contaminant by iron-based nanomaterials remains controversial. In this study, the effect of humic acid (representing NOM) on the degradation of decabromodiphenyl ether (BDE209) by biochar supported Ni/Fe nanoparticles (BC@Ni/Fe ) were investigated, which indicated that the removal of BDE209 by BC@Ni/Fe was inhibited in the presence of HA, and with the increase of HA concentration, the inhibitory effect showed more significant. The interaction between HA and BC@Ni/Fe shown that HA was quickly adsorbed on the $\mathrm{BC} @ \mathrm{Ni} / \mathrm{Fe}$. The results of the Zeta potential and sedimentation experiment of BC@Ni/Fe showed that the stability and surface charge of BC@Ni/Fe were effectively improved with the increase of HA concentration, indicating that the inhibitory effect of HA in the debromination of BDE209 by BC@Ni/Fe was not through inhibiting the performance of nanoparticles by HA. The corrosion capacity of BC@Ni/Fe decreased with the increase of HA, which did positively correlate with the effect of HA on the reactivity of BC@Ni/Fe in the removal of BDE209. Additionally, those typical quinone compounds in HA (lawsone and AQDS), which have the electron transfer function, did not serve as an electron transfer medium to directly participating in the reaction process, on the contrary, those compounds did adversely effect on the removal of BDE209. In the coexisting system of HA and BDE209, the equilibrium adsorption capacity of HA on BC@Ni/Fe was 4.75 $\mathrm{mg} / \mathrm{g}$. Conversely, the adsorption quantities of BDE209 on BC@Ni/Fe in the absence of HA was $0.31 \mathrm{mg} / \mathrm{g}$, which was about 1.3 times higher than that of in the presence of HA (the adsorption quantities of BDE209 was $0.23 \mathrm{mg} / \mathrm{g}$ ). Moreover, in the coexistent system of HA and BDE209, the kinetic rate constants for the adsorption of HA was $0.1854 \mathrm{~min}^{-1}$, which was
\end{abstract}

*E-mail: sunmoon124@163.com; zhqfang@scnu.edu.cn; Tel.: 020 39313279; Fax: 02039313279

Received February 14, 2017; published March 22, 2017.

Supporting information for this article is available free of charge via the Internet at http://sioc-journal.cn.

Project supported by Joint Foundation of NSFC-Guangdong Province (Grant No. U1401235).

项目受 NSFC-广东联合基金(No. U1401235)资助. 
approximately 45 times greater than that of BDE209 $\left(0.0041 \mathrm{~min}^{-1}\right)$. It was shown from the analyzed results that the adsorption rate of $\mathrm{HA}$ on $\mathrm{BC} @ \mathrm{Ni} / \mathrm{Fe}$ was much greater than that of BDE209. Therefore, that is to say, HA could be preferentially adsorbed onto the surface of $\mathrm{BC} @ \mathrm{Ni} / \mathrm{Fe}$. The adsorbed HA coated on the surface of $\mathrm{BC} @ \mathrm{Ni} / \mathrm{Fe}$ occupied the active sites, which hindered the nanoparticles to contact with $\mathrm{H}_{2} \mathrm{O}$, reduced the corrosion of $\mathrm{Fe}^{0}$, thus inhibited the removal of BDE209.

Keywords natural organic matter; nanoscale zero valent iron; $\mathrm{BC} @ \mathrm{Ni} / \mathrm{Fe}$; decabromodiphenyl ether; influence mechanism

\section{1 引言}

十溴联苯醚(BDE209)作为阻燃剂，在不同环境介 质中均被检测到 ${ }^{[1]}$. 由于其具有生物富集性、难降解等 特性，易对生态环境产生毒害作用 ${ }^{[2,3]}$. 因此，去除 BDE209 显得尤其重要. 零价铁, 尤其是纳米零价铁 $(\mathrm{nZVI})$ 作为一种强还原剂对卤代有机污染物有较好的 去除和转化能力 ${ }^{[4]}$. 例如, Shih 等 ${ }^{[5]}$ 用纳米零价铁去除 BDE209, 反应 40 min, BDE209 去除率达到了 90\%. 尽 管纳米零价铁能够很好的应用于多溴联苯醚的去除, 但 是与其他纳米材料类似, 纳米零价铁由于高表面能以及 本身具有磁性等特点容易团聚, 从而降低其反应活性. 为了克服纳米零价铁的缺陷, 研究者将 nZVI 负载于某 种功能材料上, 如膨润土 ${ }^{[6]}$ 、高岭土 ${ }^{[7]}$ 、树脂 ${ }^{[8]}$ 和活性 炭 ${ }^{[9]}$ 等, 能够有效地解决 nZVI 的团聚问题并提高其活 性和稳定性. 尽管采用负载固定技术可以大大提高纳米 零价铁的分散性和活性, 但是, 如何选择更好的负载材 料是今后努力的方向.

生物炭由于具有比表面积大、孔隙结构发达、来源 广泛、成本低、制备简单等特性而备受关注. 一方面, 生 物炭作为一种成本低廉的吸附剂能够有效地吸附有机 污染物和重金属 ${ }^{[10,11]}$; 再者作为载体能有效提高纳米颗 粒的分散性、稳定性, 从而提高反应活性 ${ }^{[12,13]}$. 当生物 炭和纳米颗粒两者结合时，能够发挥两者的协同效应. 例如 Devi 等 ${ }^{[14]}$ 研究发现, 生物炭负载纳米铁对五氯苯 酚(PCP)的去除率比单独的纳米零价铁和生物炭高. 此 外, $\mathrm{Su}$ 等 ${ }^{[15]}$ 采用生物炭负载纳米零价铁去除土壤中的六 价铬发现, 与纳米零价铁相比, 生物炭负载纳米铁有效 地提高了其稳定性和流动性. 因而, 生物炭是一种理想 的纳米颗粒负载载体. 与此同时, 研究发现将另外一种 贵金属(镍、钯等)沉积到生物炭负载纳米铁形成生物炭 负载纳米双金属, 能够进一步提高反应活性. 例如, Devi 等 ${ }^{[16]}$ 合成生物炭负载纳米镍铁去除 PCP, 反应 240 $\min$, 对 PCP 的去除率为 $100 \%$, 然而, 生物炭负载零价 铁对 PCP 的去除率达到 $100 \%$ 需要反应 $480 \mathrm{~min}$. Wu 等 ${ }^{[17]}$ 用生物炭负载纳米镍铁去除土壤中 BDE209, 反应 $72 \mathrm{~h}$, 对 BDE209 的去除率为 $87.7 \%$, 比单独地纳米镍铁 和生物炭分别高出 $30.2 \%$ 和 $69 \%$, 并且能够有效地减少 镍以及 BDE209 降解中间产物带来的二次污染. 因而, 制备出生物炭负载纳米双金属并用于污染物的去除则 更有价值.

天然有机质(nature organic matter, NOM) 普遍存在 于自然环境中, 具有丰富的官能团, 如羧基、酚基、羰
基、醌基等官能团，能够通过吸附、络合，以及充当电 子转移介质等作用严重影响纳米材料降解有机污染 物 ${ }^{[18,19]}$. 与此同时, 不同研究者在研究 NOM 对纳米铁 基材料去除污染物效果影响时发现, NOM 对于污染物 的去除能够起促进作用或抑制作用，存在争议. 如 Fang 等 ${ }^{[20]}$ 研究发现无论是腐殖酸(HA), 还是常用作电子转 移介质的对苯醌、2-羟基-1,4-䒺醌均没有发挥电子转移 媒介的作用, 均对 nZVI 去除 BDE209 起抑制作用; 相 反, Tratnyek 等 ${ }^{[21]}$ 发现, 醌类化合物, 例如胡桃醌、指甲 花醌、葱醌-2,6-二磺酸钠等化合物能够加速纳米零价铁 对于四氯化碳的还原降解. 另外, Doong 等 ${ }^{[2]}$ 在使用纳 米 $\mathrm{Pd} / \mathrm{Fe}$ 去除四氯乙烯时发现 HA 能够与四氯乙烯发生 竞争吸附, 使得四氯乙烯的去除率下降; 而 Kang 等 ${ }^{[23]}$ 发现, 被纳米零价铁吸附的 NOM 能够增强电子转移, 从而使得水体中溶解氧形成过氧化氢和羟基自由基, 加 速去除四氯苯酚. 尽管以上研究能够为 NOM 如何影响 纳米颗粒的环境行为提供理论依据, 但是, 如何在一个 复杂体系中更有效辨识出 NOM 对纳米铁基材料去除污 染物的主导影响作用机理，是纳米铁基材料应用于实际 环境污染修复需要解决的一个关键问题.

本工作制备出生物炭负载纳米镍铁 $(\mathrm{BC} @ \mathrm{Ni} / \mathrm{Fe})$, 以腐植酸(HA)代表天然有机质 (NOM), 主要研究了(1) $\mathrm{HA}$ 对 BC@ Ni/Fe 去除十溴联苯醚(BDE209)反应活性的 影响规律; (2) HA 对 BC@Ni/Fe 颗粒性能的影响, 包括 $\mathrm{HA}$ 对 BC@ Ni/Fe 腐蚀性能、稳定性、表面电荷的影响; (3) HA 中典型醌类代表物质对 BC@Ni/Fe 去除 BDE209 的影响; (4) BDE209 与 HA 的竞争吸附实验, 从而辨识 出 HA 对 BC@Ni/Fe 降解 BDE209 主要影响作用机制.

\section{2 结果与讨论}

\section{1 材料表征}

通过扫描电镜分析了生物炭、纳米镍铁、BC@ $\mathrm{Ni} / \mathrm{Fe}$ 的表面形态, 其结果如图 1 所示. 从图 1(a)中可以看出 生物炭成骨架状，孔隙结构发达，一方面可以为纳米颗 粒的附着提供足够的空间, 另一方面又能够利用孔道限 制纳米颗粒的团聚. 再者, 从图 1(b)可以看出, 由于本 身具有磁性以及范德华力的作用, 纳米 $\mathrm{Ni} / \mathrm{Fe}$ 成链状结 构, 团聚严重. 最后, 从图 1(c)可以看出, BC@ $\mathrm{Ni} / \mathrm{Fe}$ 中 纳米 $\mathrm{Ni} / \mathrm{Fe}$ 虽然形成链状结构, 但与纳米 $\mathrm{Ni} / \mathrm{Fe}$ 相比, 团 聚程度大大降低, 这也说明纳米 $\mathrm{Ni} / \mathrm{Fe}$ 能够较好地分散 在生物炭表面和孔隙内, 一定程度上减少了纳米颗粒的 团聚。与此同时，通过 $\mathrm{BC} @ \mathrm{Ni} / \mathrm{Fe}$ 的透射电镜图 
(TEM)(图 S1, SI)进一步得出黑色的纳米颗粒分散性良 好, 这与 $\mathrm{Wu}$ 等 ${ }^{[17]}$ 得到的研究结果类似. 此外, 负载在 生物炭上的纳米 $\mathrm{Ni} / \mathrm{Fe}$ 的平均颗粒尺寸约为 $10 \sim 80 \mathrm{~nm}$ 之间. 为了进一步确认 $\mathrm{BC} @ \mathrm{Ni} / \mathrm{Fe}$ 中各个元素的价态以 及组成情况, 采用 XPS 对 BC@ Ni/Fe 颗粒进行分析, 结 果如图 S2 (SI)所示. 通过进一步分析 Fe 的 $2 p$ 谱图时发 现, 结合能为 $707.6 \mathrm{eV}$ 表明存在零价铁, 而 $711.4 \mathrm{eV}$ 的 结合能峰表明 $\mathrm{BC} @ \mathrm{Ni} / \mathrm{Fe}$ 中铁主要以 $\mathrm{Fe}_{3} \mathrm{O}_{4}$ 或 $\mathrm{Fe}_{2} \mathrm{O}_{3}$ 的 形式存在 ${ }^{[24]}$; 同时分析 $\mathrm{Ni}$ 的 $2 \mathrm{p}$ 谱图, 表明 $\mathrm{Ni}$ 以二价态 和零价态存在 ${ }^{[24]}$. 通过上述分析说明 $\mathrm{BC} @ \mathrm{Ni} / \mathrm{Fe}$ 在表征 或制备过程中一定程度上被氧化了。
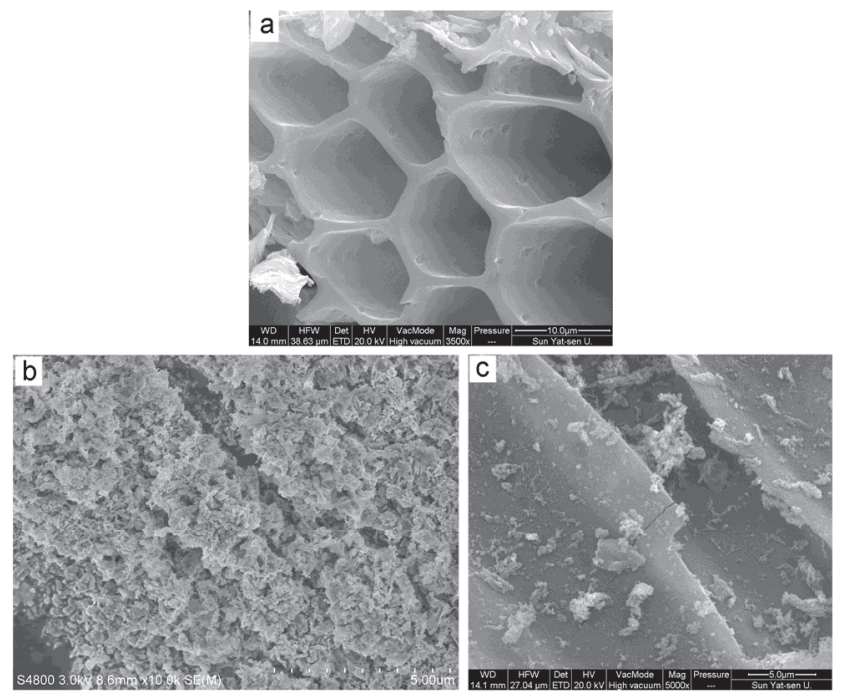

图 1 生物炭(a), 纳米镍铁(b)和生物炭负载纳米镍铁(c)的扫描电镜 图

Figure 1 SEM images of biochar(a), Ni/Fe(b) and BC@ $\mathrm{Ni} / \mathrm{Fe}(\mathrm{c})$

\section{2 腐殖酸对 BC@Ni/Fe 去除 BDE209 的影响}

不同浓度腐殖酸对 BC@Ni/Fe 去除 BDE209 的影响 如图 2 所示. 从图中可以看出, 不同浓度 $\mathrm{HA}(0 \sim 40$ $\mathrm{mg} / \mathrm{L}$ )体系中, 反应 $3 \mathrm{~h}$ 后, BC@Ni/Fe 对 BDE209的去除 率分别达到了 $100 \% 、 98.51 \% 、 83.54 \% 、 67.37 \% 、 64.36 \%$. 从结果可以看出, 虽然在不同 $\mathrm{HA}$ 浓度体系下 BC@Ni/Fe 对 BDE209 的去除率均随着反应时间的升高 而升高, 但是, 随着腐殖酸浓度升高, $\mathrm{BC} @ \mathrm{Ni} / \mathrm{Fe}$ 对 BDE209 的去除率却下降. 再者, 从反应速率常数来看 [图 2(b)], 随着 $\mathrm{HA}$ 浓度从 $0 \mathrm{mg} / \mathrm{L}$ 升高到 $40 \mathrm{mg} / \mathrm{L}$, BC@Ni/Fe 去除 BDE209 的反应速率常数也下降, 分别 对应为 $0.0173 \mathrm{~min}^{-1}(\mathrm{HA}=0 \mathrm{mg} / \mathrm{L}) 、 0.0162 \mathrm{~min}^{-1}(\mathrm{HA}=$ $5 \mathrm{mg} /) 、 0.0106 \mathrm{~min}^{-1}(\mathrm{HA}=10 \mathrm{mg} / \mathrm{L}) 、 0.0064 \mathrm{~min}^{-1}(\mathrm{HA}$ $=20 \mathrm{mg} / \mathrm{L}) 、 0.0055 \mathrm{~min}^{-1}(\mathrm{HA}=40 \mathrm{mg} / \mathrm{L})$. 因此, 腐殖 酸在 BC@Ni/Fe 去除 BDE209 起抑制作用, 而且随着 $\mathrm{HA}$ 的浓度升高, 对 BC@Ni/Fe 去除 BDE209 抑制作用 越大.
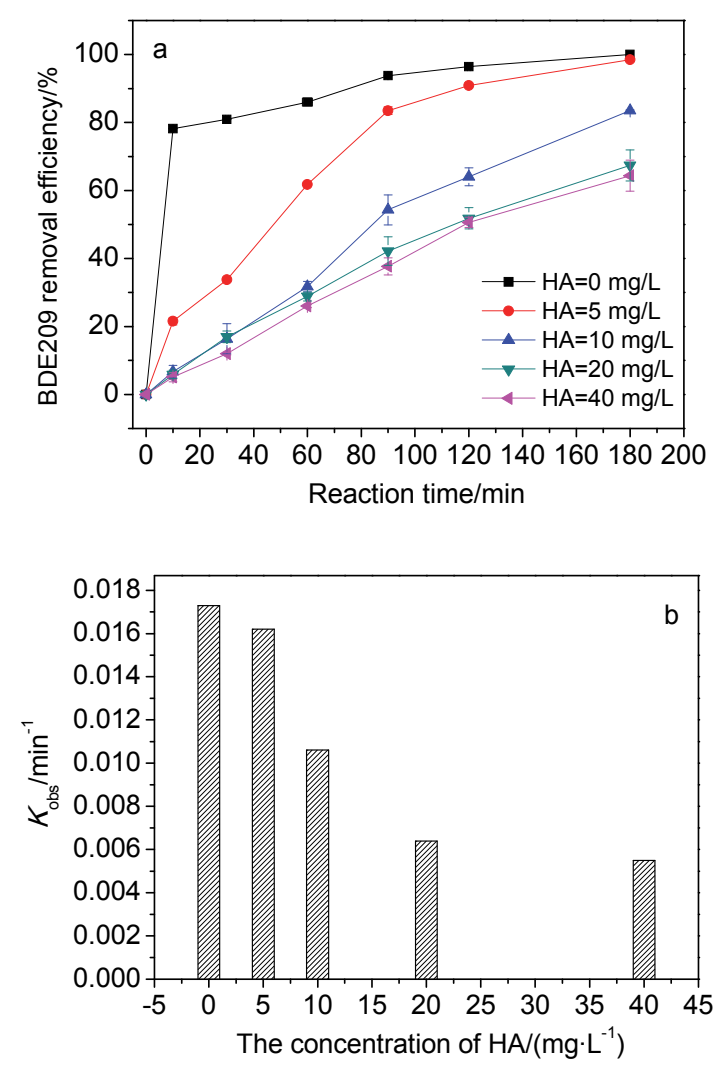

图 2 不同浓度腐殖酸对 BC@ Ni/Fe 去除 BDE209 (a) 以及反应速率常 数的影响(b)

Figure 2 Effects of the concentration of HA on the removal efficiency of BDE209 (a) and the kinetics constants (b)

值得注意的是，根据我们课题组之前文献报道，反 应 $3 \mathrm{~h}$, 未经修饰改性的纳米镍铁对 BDE209 的去除率 为 $65.48 \%{ }^{[24]}$. 但是，在相同的反应条件下，体系中无 HA 时, BC@Ni/Fe 对 BDDE209 的去除率达到了 100\%, 约为纳米镍铁的 1.5 倍. 这主要是由于 $\mathrm{BC} @ \mathrm{Ni} / \mathrm{Fe}$ 发挥 了生物炭和纳米 $\mathrm{Ni} / \mathrm{Fe}$ 的双重作用，一方面生物炭拥有 大的比表面积以及孔隙结构使得 $\mathrm{BC} @ \mathrm{Ni} / \mathrm{Fe}$ 比表面积增 大能够有效将溶液中 BDE209 吸附到材料表面, 有效地 提高了 $\mathrm{BC} @ \mathrm{Ni} / \mathrm{Fe}$ 与目标污染物的接触率 ${ }^{[17]}$; 另一方 面, 从前面的 SEM 和 TEM 的分析结果也可以看出, $\mathrm{Ni} / \mathrm{Fe}$ 能够较好地分散在生物炭上, 减少了团聚, 进一 步提高了反应活性.

尽管生物炭负载镍铁进一步提高了纳米 $\mathrm{Ni} / \mathrm{Fe}$ 的反 应活性，但是，当体系中存在 HA 时，一定程度上对 BC@Ni/Fe 去除 BDE209 起到抑制作用. 结合相关文献 以及课题组前期研究 HA 对纳米零价铁降解 BDE209 的 影响基础上, 我们认为 $\mathrm{HA}$ 在 $\mathrm{BC} @ \mathrm{Ni} / \mathrm{Fe}$ 非生物降解 BDE209 的多元体系中, 其影响作用机理主要有: (1) HA 与 BDE209 发生竞争吸附, HA 优先被 BC@Ni/Fe 快速吸 附，占据 $\mathrm{BC} @ \mathrm{Ni} / \mathrm{Fe}$ 表面的活性位点; (2) HA 对 $\mathrm{BC} @ \mathrm{Ni} / \mathrm{Fe}$ 性能的影响. 如影响 BC@Ni/Fe 的稳定性、 表面电荷、腐蚀性能; (3) HA 对反应过程的影响. 主要 
有 HA 作为电子转移介质或者作为电子转移屏障. 为此, 我们设计了相关实验来一一进行鉴别, 从而识别出 HA 对 BC@Ni/Fe 去除 BDE209 的主导影响作用机制.

\subsection{BC@Ni/Fe 去除腐殖酸}

$\mathrm{BC} @ \mathrm{Ni} / \mathrm{Fe}$ 对不同浓度的腐殖酸的去除实验结果如 图 3 所示. 从图 3(a)中可以看出, HA 能够快速地被 $\mathrm{BC} @ \mathrm{Ni} / \mathrm{Fe}$ 去除. 反应 $60 \mathrm{~min}$ 时, $\mathrm{BC} @ \mathrm{Ni} / \mathrm{Fe}$ 对不同浓度 的 HA 的去除率分别为 100\%、100\%、100\%、96.0\%; 反 应 $180 \mathrm{~min}, \mathrm{BC} @ \mathrm{Ni} / \mathrm{Fe}$ 对 $\mathrm{HA}$ 的去除率均接近 100\%. 从 实验结果来看, 当反应时间从 $60 \mathrm{~min}$ 增加到 $180 \mathrm{~min}$ 时, 不同浓度 HA 的去除率分别增长了 $0 \% 、 0 \% 、 0 \% 、 4 \%$, 说 明在 $60 \mathrm{~min} \mathrm{BC} @ \mathrm{Ni} / \mathrm{Fe}$ 对于 $\mathrm{HA}$ 的吸附已经基本达到 平衡, 且随着时间的延长 $\mathrm{HA}$ 并没有从固相解析到液相 中, 说明腐殖酸能够稳定地吸附在 $\mathrm{BC} @ \mathrm{Ni} / \mathrm{Fe}$ 的表面, 这与 Fang 等 ${ }^{[20]}$ 研究纳米零价铁去除 HA 得到结论类似. 此外, 从拟合的准一级吸附动力学(表 $\mathrm{S} 1$, 支持信息)来 看, 其拟合相关系数 $\left(R^{2}\right)$ 均大于 0.9 , 拟合度高, 说明 $\mathrm{BC} @ \mathrm{Ni} / \mathrm{Fe}$ 吸附 $\mathrm{HA}$ 符合准一级吸附动力学, 这与 Choi 等 ${ }^{[25]}$ 研究结果类似. 另外, 从 $\mathrm{HA}$ 去除结果来看说明 $\mathrm{BC} @ \mathrm{Ni} / \mathrm{Fe}$ 能够提供足够的活性位点来吸附 $\mathrm{HA}$, 但是 随着 $\mathrm{HA}$ 浓度的升高, $\mathrm{BC} @ \mathrm{Ni} / \mathrm{Fe}$ 吸附 $\mathrm{HA}$ 的速率常数降
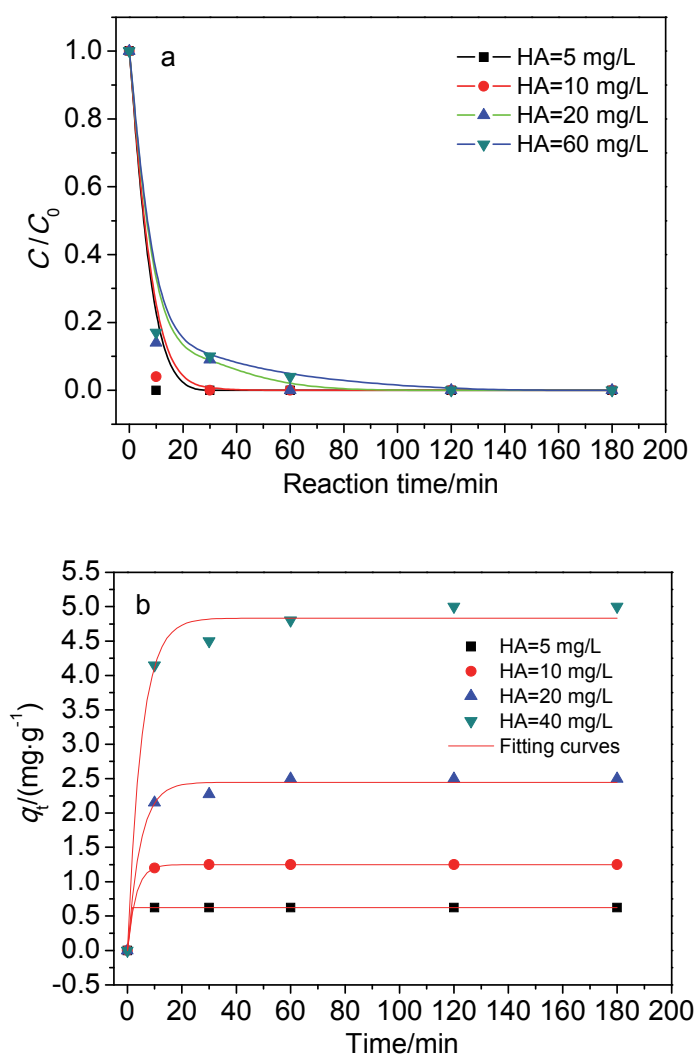

图 $33 \mathrm{BC} @ \mathrm{Ni} / \mathrm{Fe}$ 去除腐殖酸 $(\mathrm{a})$ 和 $\mathrm{BC} @ \mathrm{Ni} / \mathrm{Fe}$ 去除腐殖酸的吸附动力 学(b)

Figure 3 The removal of HA by BC@Ni/Fe (a) and adsorption kinetics of HA by BC@Ni/Fe nanoparticles (b)
低, 分别对应为 $3.6033 \mathrm{~min}^{-1}(\mathrm{HA}=5 \mathrm{mg} / \mathrm{L}) 、 0.3218$ $\min ^{-1}(\mathrm{HA}=10 \mathrm{mg} / \mathrm{L}) 、 0.2084 \min ^{-1}(\mathrm{HA}=20 \mathrm{mg} / \mathrm{L}) 、$ $0.1927 \mathrm{~min}^{-1}(\mathrm{HA}=40 \mathrm{mg} / \mathrm{L})$. 这主要是由于纳米颗粒 去除污染物属于表面反应，当 $\mathrm{BC} @ \mathrm{Ni} / \mathrm{Fe}$ 的量一定时， 提供的反应活性位点一定，随着 HA 浓度的升高，分子 间可能发生了竞争吸附，从而导致了吸附速率下降，这 与 Bokare 等 ${ }^{[26]}$ 在研究纳米镍铁去除不同初始浓度的染 料以及 Fang 等 ${ }^{[24]}$ 研究纳米镍铁去除不同浓度的 BDE209得到的结论类似. 与此同时，结合不同浓度 HA 对于 $\mathrm{BC} @ \mathrm{Ni} / \mathrm{Fe}$ 去除 $\mathrm{BDE} 209$ 的去除影响结果来看, 说 明吸附到 $\mathrm{BC} @ \mathrm{Ni} / \mathrm{Fe}$ 表面 HA 的量与 HA 浓度的升高对 $\mathrm{BC} @ \mathrm{Ni} / \mathrm{Fe}$ 去除 BDE209 反应活性影响成正相关，即 $\mathrm{BC} @ \mathrm{Ni} / \mathrm{Fe}$ 吸附 $\mathrm{HA}$ 的量越多, 对 $\mathrm{BC} @ \mathrm{Ni} / \mathrm{Fe}$ 去除 BDE209 抑制作用越显著.

\section{4 腐殖酸对 BC@Ni/Fe 稳定性影响}

不同浓度腐殖酸 $(\mathrm{HA})$ 体系中, $\mathrm{BC} @ \mathrm{Ni} / \mathrm{Fe}$ 的自由沉 降曲线如图 4(a)所示. 从图中可以看出，随着时间的延 长, 其吸光度降低越多, $30 \mathrm{~min}$ 中的时候分别降低了 $51.9 \% 、 49.1 \% 、 44.8 \% 、 41.8 \% 、 36.5 \%$, 沉降 $1 \mathrm{~h}$ 后，其 吸光度分别降低了 $64.4 \% 、 58.9 \% 、 52.7 \% 、 48.9 \% 、 44.1 \%$.
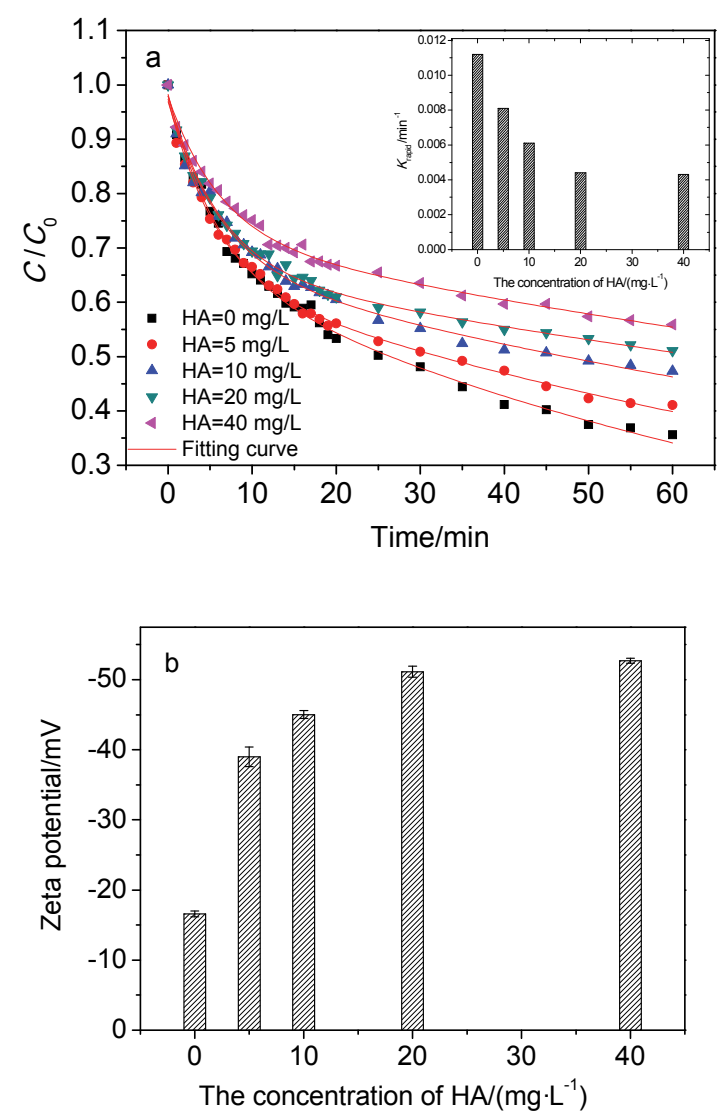

图 4 腐殖酸对 $\mathrm{BC} @ \mathrm{Ni} / \mathrm{Fe}$ 的沉降(内插图为沉降速率常数)(a)以及 zeta 电位的影响(b)

Figure 4 Effects of HA on the sedimentation (inset, settling rate constants) (a) and zeta potential (b) of BC@Ni/Fe 
另外, 不同浓度 HA 下, 通过双速率模型(biphasic rate model) 拟合 BC@Ni/Fe 的沉降速率常数结果如表 $\mathrm{S} 2$ (支 持信息)所示. 与此同时, 从图 4(a)(内插图)可以看出, 随着 HA 的浓度升高, $K_{\text {rapid }}$ (沉降速率常数)减少. 当 HA 浓度为 $0 \mathrm{mg} / \mathrm{L}$ 时, $K_{\text {rapid }}$ 为 $0.0112 \mathrm{~min}^{-1}$, HA 浓度为 20 $\mathrm{mg} / \mathrm{L}$ 时, $K_{\text {rapid }}$ 为 $0.0044 \mathrm{~min}^{-1}$, HA 浓度为 $40 \mathrm{mg} / \mathrm{L}$ 时, $K_{\text {rapid }}$ 为 $0.0043 \mathrm{~min}^{-1}$. 然而, 造成 BC@ $\mathrm{Ni} / \mathrm{Fe}$ 其吸光度 快速降低的主要原因是因为复合材料本身的磁性作用 及粒子之间的范德华力使得复合材料颗粒团聚成较大 颗粒加上自身重力作用导致沉降加快. 但是, 随着 HA 浓度的升高, 能够一定程度上减缓 $\mathrm{BC} @ \mathrm{Ni} / \mathrm{Fe}$ 的沉降. 这可能是 $\mathrm{HA}$ 被 $\mathrm{BC} @ \mathrm{Ni} / \mathrm{Fe}$ 吸附, 进一步加强了颗粒间 的静电排斥作用, 从而导致沉降速率减缓 ${ }^{[15]}$. 此外, 从 复合材料表面的 Zeta[图 4(b)]电位测定结果来看, 随着 $\mathrm{HA}$ 浓度的升高, $\mathrm{BC} @ \mathrm{Ni} / \mathrm{Fe}$ 表面电荷增大, 这也再次 说明 HA 吸附在复合材料表面会导致 BC@Ni/Fe 颗粒间 的静电排斥作用增大，从而一定程度上减缓复合材料的 沉降, 这也再次证明腐殖酸被吸附在复合材料表面. 这 与 Dong 等 ${ }^{[27]}$ 研究的结果类似.

随着 HA 浓度的升高可以有效地减缓 $\mathrm{BC} @ \mathrm{Ni} / \mathrm{Fe}$ 的 沉降速率, 从而提高其稳定性, 结合其他文献报道, 纳 米颗粒的稳定性提高, 一定程度上能够提高反应活性. 但是从前面的试验结果可知 $\mathrm{HA}$ 对 $\mathrm{BC} @ \mathrm{Ni} / \mathrm{Fe}$ 去除 BDE209 却起到抑制作用, 也就是说 HA 提高 BC@Ni/Fe 的稳定性能并不是其抑制纳米颗粒去除 BDE209 反应活 性的原因, 这与 Dong 等 ${ }^{[27]}$ 在研究 FA 对于纳米零价铁去 除六价铬得到的结果类似.

\section{5 腐殖酸对 BC@Ni/Fe 腐蚀性能影响}

天然有机质覆盖在铁基材料的表面可能影响纳米 颗粒的腐蚀性, 进而影响污染物的去除效果. 通过 $\mathrm{BC} @ \mathrm{Ni} / \mathrm{Fe}$ 析出的铁和镍离子来判别 $\mathrm{HA}$ 对 $\mathrm{BC} @ \mathrm{Ni} / \mathrm{Fe}$ 腐蚀能力影响, 其影响结果如图 5 所示. 从图 5(a)中可 以看出, 不同浓度 HA 体系中, 溶液中铁的含量均呈现 先升高后降低的趋势. 当 $\mathrm{HA}=0 \mathrm{mg} / \mathrm{L}$ 时, 在反应时间 为 $90 \mathrm{~min}$ 时, 溶液中铁的含量为 $1.680 \mathrm{mg} / \mathrm{L}$, 随着反应 的进行, 溶液中的铁含量呈现降低的趋势, 反应 180 $\min$ 时, 溶液中铁的含量降低为 $0.465 \mathrm{mg} / \mathrm{L}$. 在 $\mathrm{BC} @ \mathrm{Ni} / \mathrm{Fe}$ - 水体系中, 作为电子供体的 $\mathrm{Fe}^{0}$ 腐蚀后产 生电子并被氧化成 $\mathrm{Fe}^{2+} / \mathrm{Fe}^{3+}$, 而溶液中的水得到电子后 产生 $\mathrm{H}_{2}$ 并被零价镍活化成游离态氢原子, 使得吸附在 材料表面的 BDE209 发生加氢脱溴致使苯环上 $\mathrm{Br}-\mathrm{C}$ 键 断裂, 从而使得 BDE209 得以降解 ${ }^{[28]}$. 随着反应进行, 由于消耗了水中的质子氢, 从而导致溶液总的 $\mathrm{pH}$ 上升, 导致 $\mathrm{Fe}^{2+} / \mathrm{Fe}^{3+}$ 容易在 $\mathrm{BC} @ \mathrm{Ni} / \mathrm{Fe}$ 表面形成氢氧化物的 沉积物, 进一步导致 $\mathrm{Fe}^{0}$ 的腐蚀减慢, 与此同时的溶液 中 $\mathrm{pH}$ 的升高本身也会抑制零价铁的腐蚀, 从而导致溶 液中铁的含量降低 ${ }^{[8,29]}$.
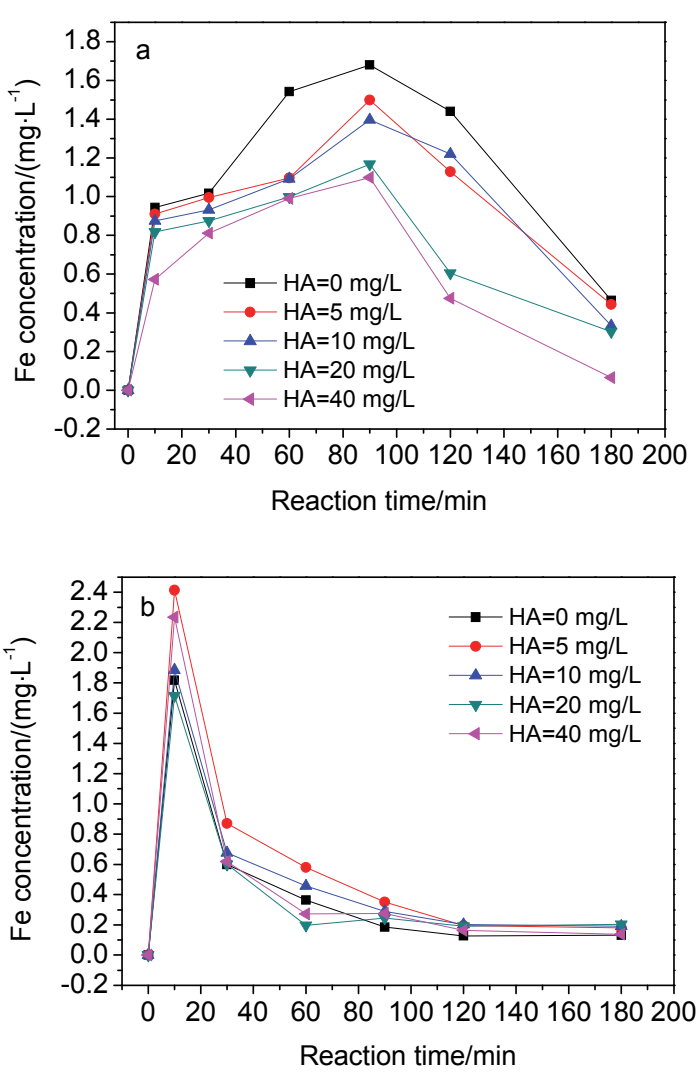

图 $5 \mathrm{BC} @ \mathrm{Ni} / \mathrm{Fe}$ 在不同浓度腐殖酸体系中铁离子 $(\mathrm{a})$ 和镍离子 $(\mathrm{b})$ 的析 出量

Figure 5 The dissolved iron (a) and nickle (b) content leaching from the BC@Ni/Fe nanoparticles under different concentration of HA

此外, 由于生物炭的作用也能够有效地吸附铁离 子 ${ }^{[17,30]}$. 例如 $\mathrm{Su}$ 等 ${ }^{[30]}$ 合成了生物炭负载零价铁去除土 壤中六价铬, 与单纯的纳米零价铁相比, 复合材料有效 地减少了土壤中有效铁含量, 同样地 Devi 等 ${ }^{[14]}$ 合成生 物炭负载零价铁去除 PCP 也有效地减少了铁的析出. 与 此同时, HA 本身具有复杂的结构, 含有很多官能团例 如羧基和酚类、硫酸盐、磷酸盐和氨基酸, 也能够有效 地吸附螯合金属离子，从而导致溶液中的铁离子含量减 少. 此外, 从图 5(a)中可以看出体系中存在 HA 时, 不同 浓度 HA 体系溶液中铁的含量随着反应时间的上升，均 呈现出先升高后下降的趋势. 随着 HA 浓度的升高, 溶 液中的铁的含量在不同反应时间点均呈下降趋势，而且 HA 浓度越高, 铁的含量越低, 反应结束时, 溶液中铁 的含量分别为 $0.444 \mathrm{mg} / \mathrm{L}(\mathrm{HA}=5 \mathrm{mg} / \mathrm{L}) 、 0.333 \mathrm{mg} / \mathrm{L}$ $(\mathrm{HA}=10 \mathrm{mg} / \mathrm{L}) 、 0.303 \mathrm{mg} / \mathrm{L}(\mathrm{HA}=20 \mathrm{mg} / \mathrm{L}) 、 0.066 \mathrm{mg} / \mathrm{L}$ $(\mathrm{HA}=40 \mathrm{mg} / \mathrm{L})$. 这与前面的分析结果, 腐殖酸对 $\mathrm{BC} @ \mathrm{Ni} / \mathrm{Fe}$ 去除 BDE209 起抑制作用, 而且随着 HA 的 浓度升高, 抑制作用越显著的趋势一致. 由于纳米铁基 材料具有强还原性, 一定程度上 $\mathrm{Fe}^{0}$ 腐蚀的越快, 产生 的电子越多, 越有利于加快反应, 溶液中的铁含量也相 对升高. Xie 等 ${ }^{[31}$ 研究发现, 随着腐殖酸浓度的升高, 纳 米零价铁对于溴酸盐的去除率分别减小了 $1.3 \sim 2.0$ 倍, 
并且认为造成去除效率下降的原因是腐殖酸迅速吸附 到纳米零价铁的表面并且快速与不同类型的铁 $\left(\mathrm{Fe}^{0} / \mathrm{Fe}^{2+}\right.$ $/ \mathrm{Fe}^{3+}$ )形成络合物, 使纳米零价铁表面钝化. 再者腐殖 酸与 $\mathrm{Fe}^{2+}$ 形成的络合物也会阻碍 $\mathrm{Fe}^{2+}$ 对溴酸盐的还原 或者是占据活性位点和阻碍铁的腐蚀, 从而导致溴酸盐 的去除率下降. Zhang 等 ${ }^{[32]}$ 研究 HA 对纳米镍铁去除 2,4二氯苯酚的影响发现, 溶液中铁含量先升高后下降, HA 吸附于纳米镍铁表面影响了纳米镍铁的腐蚀, 进而对纳 米 $\mathrm{Ni} / \mathrm{Fe}$ 去除 2,4-二氯苯酚起到抑制作用. 前面的分析 已经表明 $\mathrm{BC} @ \mathrm{Ni} / \mathrm{Fe}$ 能够快速地将腐殖酸从水溶液中吸 附到 BC@Ni/Fe 的表面. 因此, HA 吸附在 BC@Ni/Fe 的 表面会影响到 $\mathrm{BC} @ \mathrm{Ni} / \mathrm{Fe}$ 的腐蚀能力, 进一步影响了 BC@Ni/Fe 去除 BDE209.

此外, 溶液中镍离子的析出情况如图 5(b)所示. 从 图中可以看出随着反应进行, 不同浓度 HA 体系溶液中 镍离子的浓度随着反应时间的上升, 呈现先升高后下降 的趋势. 反应 $10 \mathrm{~min}$ 时, 不同浓度 $\mathrm{HA}$ 体系中镍离子的 浓度分别为 $1.818 \mathrm{mg} / \mathrm{L} 、 2.413 \mathrm{mg} / \mathrm{L} 、 1.886 \mathrm{mg} / \mathrm{L} 、 1.715$ $\mathrm{mg} / \mathrm{L} 、 2.235 \mathrm{mg} / \mathrm{L}$, 值得注意的是, 溶液中的镍的含量 均已经超过污水排放标准中镍离子 $(1 \mathrm{mg} / \mathrm{L})$ 的最高排放 标准(GB8978-1996, 中国), 但是, 反应结束之后, 不同 $\mathrm{HA}$ 体系溶液中的镍离子浓度分别为 $0.132 \mathrm{mg} / \mathrm{L} 、 0.181$ $\mathrm{mg} / \mathrm{L} 、 0.192 \mathrm{mg} / \mathrm{L} 、 0.204 \mathrm{mg} / \mathrm{L} 、 0.138 \mathrm{mg} / \mathrm{L}$, 均未超过 污水排放标准中镍离子 $(1 \mathrm{mg} / \mathrm{L})$ 的最高排放标准 (GB8978-1996, 中国). 然而, 课题组前期利用纳米镍铁 去除 BDE209 反应 $3 \mathrm{~h}$ 后, 溶液中镍离子的含量为 4.48 $\mathrm{mg} / \mathrm{L}^{[24]}$. 而造成溶液中镍离子浓度减少的原因可能为 一方面生物炭能够有效地吸附螯合镍离子; 另一方面随 着 $\mathrm{HA}$ 浓度的升高, $\mathrm{HA}$ 吸附并包覆在 $\mathrm{BC} @ \mathrm{Ni} / \mathrm{Fe}$ 表面, 阻碍了 $\mathrm{BC} @ \mathrm{Ni} / \mathrm{Fe}$ 与水有效接触, 不仅抑制了铁的析出 也抑制了镍离子的析出.

\section{6 醌类物质对 BC@Ni/Fe 去除 BDE209 的影响}

虽然腐殖酸快速的被 $\mathrm{BC} @ \mathrm{Ni} / \mathrm{Fe}$ 吸附导致占据了 $\mathrm{BC} @ \mathrm{Ni} / \mathrm{Fe}$ 表面的活性位点抑制反应的进行, 但是腐殖 酸内含有可充当电子转移媒介醌类物质, 有可能加速目 标污染物的去除 ${ }^{[20,21]}$. 从前面的 HA 对 $\mathrm{BC} @ \mathrm{Ni} / \mathrm{Fe}$ 去除 BDE209 的影响分析结果来看, 我们推断存在两种情况: 一是, 腐殖酸中的醌类化合物在反应过程中根本没有起 到电子转移媒介的作用; 二是, 虽然腐殖酸中的醌类化 合物在反应过程起到电子转移媒介作用, 但是 HA 吸附 在 $\mathrm{BC} @ \mathrm{Ni} / \mathrm{Fe}$ 的表面占据活性位点抑制作用远远大于 HA 中醌类化合物在反应过程中充当电子转移媒介的作 用. 因此, 我们研究了腐殖酸中典型的具有电子转移媒 介的醌类化合物代表 Lawsone 和 AQDS 对 BC@Ni/Fe 去除 BDE209 的影响. 从图 6 中可以看出, 与腐殖酸的 影响情况类似, Lawsone 对 BDE209 的去除起抑制作用, 而且随着浓度的升高, 抑制作用越明显, 反应结束时, 体系中不含 Lawsone 的 BC@Ni/Fe 对 BDE209 的去除率
(100\%) 分别约为不同浓度 Lawsone 体系中的 83.64\% (5 $\mathrm{mg} / \mathrm{L}) 、 70.35 \%$ (10 mg/L)、55.75\% (20 mg/L)、44.53\% (40 $\mathrm{mg} / \mathrm{L})$. 从分析结果来看 Lawsone 非但没有在 BC@ $\mathrm{Ni} / \mathrm{Fe}$ 去除 BDE209的反应中发挥电子转移媒介的作用加速目 标污染物的去除, 相反 Lawsone 对于 $\mathrm{BC} @ \mathrm{Ni} / \mathrm{Fe}$ 去除 BDE209 的抑制作用比同浓度 HA 大. 研究表明, 醌类 化合物能发挥电子转移作用的前提是电子供体能与醌 发生可逆的氧化还原反应，生成如半醌自由基、氢醌等 活性组分. 但是，当醌参与不可逆的单边反应后，会产 生一系列不具备醌特性的产物，导致其失去活性. 由于 $\mathrm{BC} @ \mathrm{Ni} / \mathrm{Fe}$ 具有强还原性, 可能会与 Lawsone 发生单边 的还原反应，生成不具备催化活性的产物，从而不能发 挥电子转移媒介的作用, 在醌的还原过程中, 它反而会 与 BDE209 争夺铁腐蚀产生的电子, 使 BDE209 的还原 速率受到抑制 ${ }^{[20]}$. 此外, 从图 6(b) 可以看出相对于不含 AQDS 体系而言, AQDS 对于 BC@Ni/Fe 去除 BDE209 起抑制作用，而且随着 AQDS 的浓度升高，抑制作用越 显著，反应结束后，对 BDE209 的去除率分别为 $93.51 \%$ (5 mg/L)、79.65\% (10 mg/L)、59.37\% (20 mg/L)、49.63\% $(40 \mathrm{mg} / \mathrm{L})$. 从结果来看, AQDS 对于 $\mathrm{BC} @ \mathrm{Ni} / \mathrm{Fe}$ 去除 BDE209 的抑制作用小于 Lawson，导致这种现象的主要 原因可能是由于两种醌类的化学结构式不同引起的.
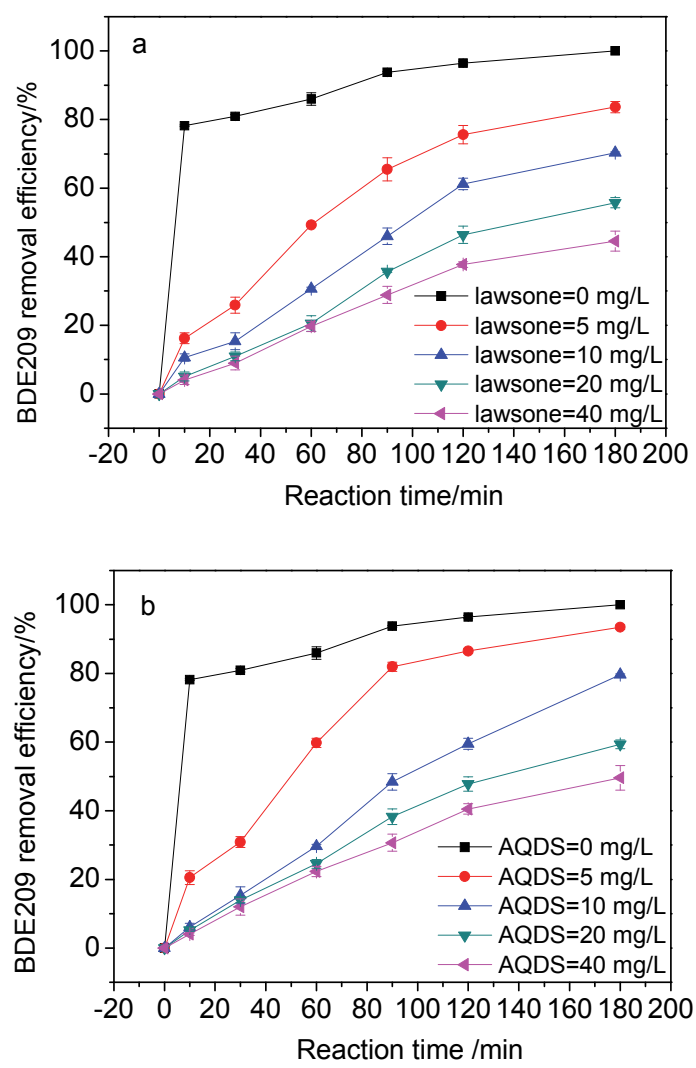

图 6 Lawsone (a)以及 AQDS (b)对 BC@Ni/Fe 去除 BDE209 的影响 Figure 6 Effects of Lawsone (a) and AQDS (b) on the removal of BDE209 by BC@Ni/Fe 
因此, $\mathrm{HA}$ 中典型的醌类化合物对 $\mathrm{BC} @ \mathrm{Ni} / \mathrm{Fe}$ 去除 BDE209 未起到电子传递的作用提高反应速率, 反而起 到抑制作用.

\subsection{HA 影响 BC@Ni/Fe 去除 BDE209 的作用机制}

纳米铁基材料在液相中去除有机污染物的过程属 于非均相反应, 存在着吸附过程和还原降解过程, 即纳 米材料先快速的将目标污染物吸附到表面, 随后立即发 生还原降解并且这个反应过程是瞬时的. Devi 等 ${ }^{[14]}$ 研究 生物炭负载纳米镍铁去除 PCP 时认为, PCP 首先快速地 吸附到生物炭负载纳米镍铁表面, 随后立即发生还原降 解, 吸附的速率决定整个反应速率. 同样地, Choi 等 ${ }^{[33]}$ 用活性炭负载纳米 $\mathrm{Fe} / \mathrm{Pd}$ 去除 PCBs, 发现 PCBs 首先快 速并全部被材料吸附固定, 接着同时发生降解, 并认为 吸附速率的快慢会影响整个反应过程. 同样的 Zhang 等 ${ }^{[35]}$ 发现 BDE21 先快速的被活性炭负载钯铁吸附, 随 后快速发生降解. 而发生还原降解的原因主要为 $\mathrm{Fe}^{0}$ 与 溶液中的 $\mathrm{H}_{2} \mathrm{O}$ 反应产生质子氢, 质子氢在贵金属催化作 用下形成原子氢，吸附在纳米铁基材料表面的卤化物中 的卤元素被氢替代 ${ }^{[24,29,33,34,35]}$. 接下来, $\mathrm{Fe}^{2+}$ 被氧化成 $\mathrm{Fe}^{3+}$, 与氢氧根反应沉积到纳米颗粒表面, 进一步阻碍 反应的进行. Zhang 等 ${ }^{[35]}$ 认为 $\mathrm{Fe}^{0}$ 的腐蚀效率会影响整个 反应过程从而影响污染物的去除. 由于纳米铁基材料具 有选择性差的特点, 当体系中存在多种物质时, 由于不 同物质之间理化性质的不同，必然导致 $\mathrm{BC} @ \mathrm{Ni} / \mathrm{Fe}$ 的环 境行为发生变化. 结合我们之前的实验结果可知, 首先, $\mathrm{HA}$ 提高了 $\mathrm{BC} @ \mathrm{Ni} / \mathrm{Fe}$ 的稳定性和表面电荷量, 但是并 没有提高 BC@Ni/Fe 对 BDE209 去除效率. 其次, HA 抑 制了 $\mathrm{BC} @ \mathrm{Ni} / \mathrm{Fe}$ 的腐蚀. 最后, 吸附在 BC@Ni/Fe 表面 的 $\mathrm{HA}$ 也没有起到电子转移媒介作用加速反应的进行. 因此, 我们将 HA 对于 BC@Ni/Fe 去除 BDE209 的影响 机制进一步缩小为 HA 与 BDE209发生竞争吸附, 即 HA 优先被 $\mathrm{BC} @ \mathrm{Ni} / \mathrm{Fe}$ 吸附, 包覆在 $\mathrm{BC} @ \mathrm{Ni} / \mathrm{Fe}$ 的表面, 占 据了 $\mathrm{BC} @ \mathrm{Ni} / \mathrm{Fe}$ 的表面活性位点, 影响了 $\mathrm{BC} @ \mathrm{Ni} / \mathrm{Fe}$ 与 水接触, 减少了其腐蚀, 从而导致了对 BDE209 的去除 效果下降.

为此, 我们分别设计了 $\mathrm{BC} @ \mathrm{Ni} / \mathrm{Fe}$ 对单独 $\mathrm{HA}$, BDE209 的去除, 以及 HA 和 BDE209 共存体系中 $\mathrm{BC} @ \mathrm{Ni} / \mathrm{Fe}$ 分别去除 HA 和 BDE209 的实验, 其中 HA 的浓度为 $40 \mathrm{mg} / \mathrm{L}$, 并采用准一级吸附动力学进行拟合 结果如图 7 所示, 相关拟合的参数见表 S3(支持信息). 从图中可以看出, 无论在何种体系下, $\mathrm{BC} @ \mathrm{Ni} / \mathrm{Fe}$ 能够 快速地吸附 HA, 反应 $3 \mathrm{~h}$, 对 $\mathrm{HA}$ 的去除率均接近 $100 \%$; 相反地，反应 $3 \mathrm{~h}$, 体系中无 HA 时 BC@ Ni/Fe 对 BDE209 的去除率为 $100 \%$, 而体系中存在 HA 时 $\mathrm{BC} @ \mathrm{Ni} / \mathrm{Fe}$ 对 BDE209 去除率为 64.36\%. 另外, 从表 $\mathrm{S} 1$ (支持信息)中可以看出, BC@Ni/Fe 对单独的 $\mathrm{HA}$ 的平 衡吸附量为 $4.83 \mathrm{mg} / \mathrm{g}$, 其次, 在 HA 和 BDE209 共存体 系中, $\mathrm{BC} @ \mathrm{Ni} / \mathrm{Fe}$ 对 $\mathrm{HA}$ 的平衡吸附量为 $4.75 \mathrm{mg} / \mathrm{g}$, 从分
析结果来看 $\mathrm{BC} @ \mathrm{Ni} / \mathrm{Fe}$ 对 $\mathrm{HA}$ 的吸附量几乎不受 BDE209 的影响. 相反地, BC $@ N$ Ni/Fe 对 BDE209 的吸附 量为 $0.31 \mathrm{mg} / \mathrm{g}$, 约为在 HA 体系下对 BDE209 的平衡吸 附量 $(0.23 \mathrm{mg} / \mathrm{g})$ 的 1.34 倍. 此外, 在 HA 和 BDE209 共 同存在下, $\mathrm{BC} @ \mathrm{Ni} / \mathrm{Fe}$ 对 $\mathrm{HA}$ 的吸附速率常数为 0.1854 $\mathrm{min}^{-1}$ 约为对 BDE209 的吸附速率常数 $\left(0.0041 \mathrm{~min}^{-1}\right)$ 的 45 倍, 也就是说 $\mathrm{BC} @ \mathrm{Ni} / \mathrm{Fe}$ 能够优先吸附 HA.
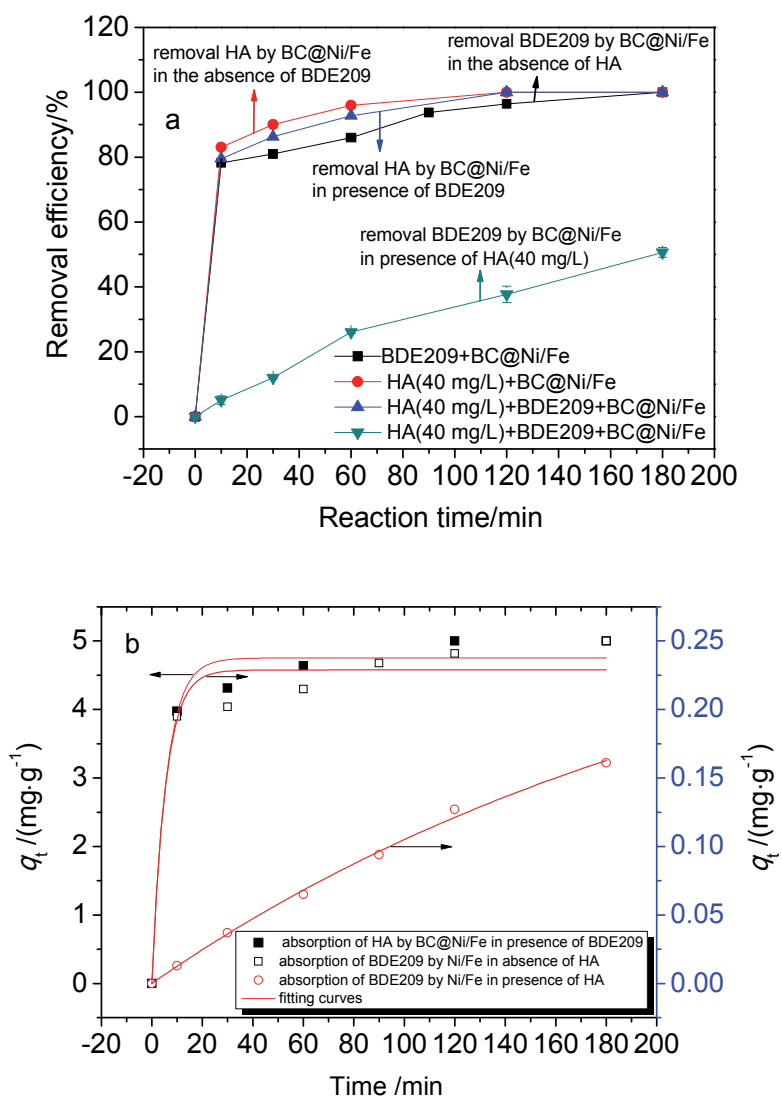

图 7 (a) HA 与 BDE209 的竞争吸附实验; (b) BC@Ni/Fe 对 BDE209 和腐殖酸的吸附动力学

Figure7 (a) Competitive adsorption of BDE209 and HA; (b) Adsorption kinetics of HA, BDE209 by BC@Ni/Fe

因此, 综合以上分析, $\mathrm{HA}$ 对 $\mathrm{BC} @ \mathrm{Ni} / \mathrm{Fe}$ 去除 BDE209 产生抑制作用的机理是: HA 与 BDE209发生了 竞争吸附, 即 $\mathrm{HA}$ 快速地被吸附包裹在 $\mathrm{BC} @ \mathrm{Ni} / \mathrm{Fe}$ 的表 面占据了活性位点, 影响了 $\mathrm{BC} @ \mathrm{Ni} / \mathrm{Fe}$ 与水的有效接触, 减少了 $\mathrm{Fe}^{0}$ 的腐蚀, 从而抑制了 $\mathrm{BC} @ \mathrm{Ni} / \mathrm{Fe}$ 对 $\mathrm{BDE} 209$ 的去除.

\section{3 结论}

本文研究了 HA 对于 BC@Ni/Fe 去除 BDE209 的影 响作用机理. 实验表明, HA 投加量越多, 对 BC@Ni/Fe 去除 BDE209 的抑制作用越显著. 在 HA 存在下, HA 与 BDE209 发生了竞争吸附, HA 优先吸附于 BC@Ni/Fe 表 面，占据了 $\mathrm{BC} @ \mathrm{Ni} / \mathrm{Fe}$ 表面的活性位点，抑制了 
$\mathrm{BC} @ \mathrm{Ni} / \mathrm{Fe}$ 的腐蚀，从而抑制了 $\mathrm{BC} @ \mathrm{Ni} / \mathrm{Fe}$ 对 BDE209 的去除. 因此, BC@Ni/Fe 在实际环境修复时, 需要考虑 天然有机质的影响.

\section{4 实验部分}

材料的制备与表征、试剂、相关的实验内容以及相 关步骤见支持信息(Supporting Information)中.

\section{References}

[1] Cheng, Z. N.; Wang, Y.; Wang, S. R.; Luo, C. L.; Li, J.; Chaemfa, C.; Jiang, H. Y.; Zhang, G. Environ. Pollut. 2014, 191, 126.

[2] Labadie, P.; Tlili, K.; Alliot, F.; Bourges, C.; Desportes, A.; Chevreuil, M. Anal. Bioanal. Chem. 2010, 396, 865.

[3] Leal, J. F.; Esteves, V. I.; Santos, E. B. H. Environ. Sci. Technol. 2013, 47, 14010

[4] Li, J. X.; Qin, H. J.; Zhang, X. Y.; Guan, X. H. Acta Chim. Sinica 2017, 75, 544 (in Chinese). (李锦祥, 秦荷杰, 张雪莹, 关小红, 化 学学报, 2017, 75, 544).

[5] Shih, Y. H.; Tai, Y. T. Chemosphere 2010, 78, 200

[6] Lin, Y. M.; Chen, Z. X.; Chen, Z. L.; Megharaj, M.; Naidu, R. Appl. Clay Sci. 2014, 93, 56.

[7] Zhang, X.; Lin, S.; Lu, X. Q.; Chen, Z. L. Chem. Eng. J. 2010, 163, 243.

[8] Li, A.; Tai, C.; Zhao, Z. S.; Wang, Y. W.; Zhang, Q. H.; Jiang, G. B.; Hu, J. T. Environ. Sci. Technol. 2007, 41, 6841 .

[9] Wu, X. Q.; Yang, Q.; Xu, D. C.; Zhong, Y.; Luo, K.; Li, X. M.; Chen, H. B.; Zeng, G. M. Ind. Eng. Chem. Res. 2013, 52, 12574.

[10] Luo, L.; Luo, L. P.; Cui, X.; Wu, B.; Hou, J.; Xun, B.; Xu, X.; Chen, Y. J. Hazard. Mater. 2011, 185, 639.

[11] Wang, X. L.; Xing, B. S. Environ. Sci. Technol. 2007, 41, 8342.

[12] Zhou, Y.; Gao, B.; Zimmerman, A. R.; Chen, H.; Zhang, M.; Cao, X. Bioresour. Technol. 2014, 152, 538.

[13] Yao, Y.; Gao, B.; Chen, J. J.; Zhang, M.; Inyang, M.; Li, Y. C.; Alva, A.; Yang, L. Y. Bioresour. Technol. 2013, 138, 8.
[14] Devi, P.; Saroha, A. K. Bioresour. Technol. 2014, 169, 525.

[15] Su, H. J.; Fang, Z. Q.; Tsang, P. E.; Fang, J. Z.; Zhao, D. Y. Environ. Pollut. 2016, 214, 94.

[16] Devi, P.; Saroha, A. K. Chem. Eng. J. 2015, 271, 195.

[17] Wu, J.; Yi, Y. Q.; Li, Y. Q.; Fang, Z. Q.; Tsang, P. E. J. Hazard. Mater. 2016, 320, 341

[18] Wang, Y. J.; Xiao, H. L.; Wang, F. Sciences in Cold and Arid Regions 2009, 1, 0372 .

[19] Smith, D. S.; Wu, F. C. Appl. Geochem. 2007, 22, 1567.

[20] Tan, L.; Liang, B.; Fang, Z. Q.; Xie, Y. Y.; Tsang, E. P. J. Nanopart Res. 2014, 162, 786.

[21] Tratnyek, P. G.; Scherer, M. M.; Deng, B. L.; Hu, S. D. Water Res. 2001, 35, 4435 .

[22] Doong, R. N.; Lai, Y. J. Water Res. 2005, 39, 2309

[23] Kang, S. H.; Choi, W. Y. Environ. Sci. Technol. 2009, 43, 878.

[24] Fang, Z. Q.; Qiu, X. H.; Chen, J. H.; Qiu, X. Q. J. Hazard. Mater. 2011, 185,958 .

[25] Giasuddin, A. M.; Kanel, S.; Choi, H. Environ. Sci. Technol. 2007, $41,2022$.

[26] Bokare, A. D.; Chikate, R. C.; Rode, C. V.; Paknikar, K. M. Appl. Catal., B 2008, 79, 270.

[27] Dong, H. R.; Ahmad, K.; Zeng, G. M.; Li, Z. W.; Chen, G. Q.; He Q.; Xie, Y. K.; Wu, Y. N.; Zhao, F.; Zeng, Y. L. Environ. Pollut. 2016, 211, 363.

[28] Xie, Y. Y.; Fang, Z. Q.; Cheng, W.; Tsang, P. E.; Zhao, D. Y. Sci. Total Environ. 2014, 485, 363

[29] Xie, Y. Y.; Fang, Z. Q.; Qiu, X. H.; Tsang, E. T.; Liang, B. Chemosphere 2014, 108, 433.

[30] Su, H. J.; Fang, Z. Q.; Tsang, P. E.; Zheng, L. C.; Fang, J. Z.; Fang, J. Z.; Zhao, D. Y. J. Hazard. Mater. 2016, 138, 533.

[31] Xie, L.; Shang, C. Environ. Sci. Technol. 2005, 39, 1092.

[32] Zhang, Z.; Cissoko, N.; Wo, J. J.; Xu, X. H. J. Hazard. Mater. 2009, 165,78 .

[33] Choi, H.; Al-Abed, S. R.; Agarwal, S.; Dionysiou, D. D. Chem. Mater. 2008, 20, 3649.

[34] Zhuang, Y.; Ahn, S.; Seyfferth, A. L.; Yoko, M. S.; Scott, F.; Richard, G. L. Environ. Sci. Technol. 2011, 45, 4896.

[35] Zhang, Z.; Cissoko, N.; Wo, J. J.; Xu, X. H. J. Hazard. Mater. 2010, $182,252$.

(Cheng, B.) 